\title{
COMMENTARY
}

\section{Call for a pan-Canadian approach to ethics review in Canada}

\author{
Stuart G. Nicholls PhD, Karine Morin LLM, Laurel Evans LLB, Holly Longstaff PhD
}

Cite as: CMAJ 2018 May 7;190:E553-5. doi: 10.1503/cmaj.171088

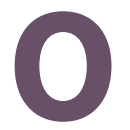

btaining research ethics approval is an important component of research conducted in humans. Increasingly, researchers undertake multicentre studies, which can require them to seek approvals from a number of research ethics boards (REBs). Many researchers have complained about variability in decisions made by REBs, as well as long delays and increased costs associated with obtaining ethics approval. Some attempts have been made to reduce variation and delays but, despite many reports and intentions to make processes of research ethics approval in Canada more efficient, no national framework for REBs reviewing health research has been achieved. Independent provincial initiatives have arisen, yet obtaining ethics review for the conduct of national and international multicentre studies remains difficult. To truly harmonize ethics review in Canada, national leadership is required to drive change that is both evidence-based and responsive to criticisms.

The purpose of research ethics review is to protect human participants involved in research while ensuring that the level of scrutiny of the proposed project is consistent with the level of risk it poses to participants. ${ }^{1}$ Some have argued that REBs impede potentially beneficial research - particularly collaborative multicentre research - through variation in the requirements of individual boards, slow processes and the requirement for duplication of effort on the part of researchers. In Canada, institutional REBs have developed independently of one another and, despite attempts to harmonize guidance on the ethical conduct and review of health research, there is no all-encompassing guidance for health-related research: the 1998 Tri-Council Policy Statement, and subsequent revisions, relates only to research conducted at institutions funded by the three major granting agencies or an organization that has adopted the policy statement as a matter of course. ${ }^{2}$ The Canadian General Standards Board standards for REBs that assess biomedical clinical trials are voluntary and focused on Health Canada-regulated clinical trials reviewed and conducted under the Good Clinical Practices section in part C, division 5 of the Canadian Food and Drug Regulations. ${ }^{3}$ Furthermore, provincial privacy legislation is often cited as a barrier for interprovincial acceptance of ethics reviews. ${ }^{4}$

\section{KEY POINTS}

- There is currently variation across Canada with regard to the structure and implementation of ethics review of research conducted in humans.

- No overarching national framework exists to streamline and enhance the quality of ethics review of human health research.

- Accreditation has been proposed, but this approach is flawed.

- There is a need for national leadership to work with provinces, develop a culture of collaboration and ensure the development of an evidence base to guide the organization and conduct of ethics review.

Some countries, such as New Zealand, the United States and the United Kingdom, have sought to solve similar problems through a process of accrediting REBs. Although approaches vary, ${ }^{5}$ the idea is that accreditation will harmonize REB operations by requiring them to meet a particular set of procedural standards. The intended result is more consistent reviews through standardized procedures, and less duplication of effort and expense. Although this approach has been discussed and touted many times in Canada (Appendix 1, available at www.cmaj.ca/lookup/suppl/doi:10.1503/cmaj. $171088 /-/ D C 1)$, we believe that accreditation of REBs is problematic.

First, many of the requirements imposed by accreditation have little or no impact on REBs' ability to protect research participants or on measures of performance, including quality of review. ${ }^{6-8}$ Second, REBs already have the ability to become accredited by the US-based Association for the Accreditation of Human Research Protection Programs. Yet few Canadian ethics boards have pursued this option owing to lack of resources and financial support. Third, provinces have now proceeded independently, focusing on provincial harmonization initiatives, which can be categorized broadly as follows: centralized review with varying board of record, in which there are reciprocal arrangements between REBs to accept each other's reviews; centralized review with a single 
board of record, in which a review is conducted by an external or multi-institutional REB; collaborative review, in which the initial review receives input from other relevant boards; and consolidation initiatives, in which boards have been aggregated to reduce the number within a province or region (Appendix 2, available at www.cmaj.ca/lookup/suppl/doi: 10.1503/cmaj.171088/-/DC1). Given the substantial investments of time and resources in these initiatives, it is unlikely that they would be revoked and replaced by a new accreditation process with its associated costs. Yet despite these investments, problems still arise when investigators from different provinces hope to collaborate on a project across provincial jurisdictions.

We suggest that the best solution for Canada would be to develop a national leadership body to work with provincial initiatives and develop national cooperation and support, facilitating acceptance of reviews between provinces. This should be supported by ongoing efforts to gather evidence of best practices on which to design, implement and evaluate future changes.

Similar proposals have come from several commissioned working groups, ${ }^{9}$ although these proposals have yet to be realized. ${ }^{8}$ Whereas bodies such as Health Canada and the tricouncils have played a role in bringing groups together, no consistent leadership has emerged to advance the governance of ethics review in Canada beyond the publication of reports. There is an urgent need to identify leadership that will champion, shape and direct the development of research ethics across Canada, in close collaboration with REBs and other research stakeholders (including researchers, research institutions, funding agencies and regulators). Although the Canadian Association of Research Ethics Boards (which represents REBs across Canada) and the Interagency Advisory Panel on Research Ethics (PRE) represent national bodies that might be seen as contenders to take forward this agenda, both have mandates that extend beyond health research. Furthermore, PRE is limited in scope because its mandate emanates from the federal funding agencies and extends only to institutions that receive such funds.

An independent leadership body that represents all key stakeholders in ethics review must be formed to engage national and provincial bodies and develop a pan-Canadian framework that benefits all research stakeholders. Given the existing provincial initiatives, engagement would likely revolve around developing equivalencies between initiatives to facilitate acceptance of ethics reviews across institutions and provincial borders. Provinces could retain their own program, obviating the need for costly reassessment of REBs under an all-encompassing accreditation standard, and honouring investments already made in these efforts, while still acknowledging the need for comparability and reciprocity. Facilitated by the proposed leadership body, REBs could be encouraged to share expertise or experience with online submission systems, or researchers could share positive experiences regarding aspects of review that were seen as beneficial. A centralized body could also provide an inventory of REBs engaged in health research, and the number of studies reviewed, information that is currently nearly impossible to obtain.

Strong leadership, harmonized provincial practices and the development of a culture of collaboration could also ensure the development of an evidence base to guide the organization and conduct of ethics review. Basic data regarding staffing, number and types of studies reviewed, timelines for review and review determinations could be captured routinely; longitudinal data would allow for identification of trends or cases that prove to be outliers. Yet there remains a dearth of data regarding the strengths or weaknesses of the different provincial approaches, partly because of a lack of consensus regarding which data should be collected - even though there are at least two systematic reviews that have identified a number of possible measures ${ }^{10,11}$ - and partly owing to varying definitions for seemingly straightforward metrics. ${ }^{12}$ Identifying similarities or differences on a set of core data elements would facilitate the sharing of best practices and help foster a culture of evaluation.

A national leadership body dedicated to the harmonization of human health research ethics review is the best solution to improve research ethics approval, particularly for multicentre, cross-provincial Canadian studies, and will facilitate development of an evidence base to inform ongoing change to improve the health research environment in Canada.

\section{References}

1. Canadian Institutes of Health Research; Natural Sciences and Engineering Research Council of Canada; Social Sciences and Humanities Research Council of Canada. Tri-council policy statement: ethical conduct for research involving humans. Ottawa: Interagency Secretariat on Research Ethics; 2010.

2. Lemmens T. Federal regulation of REB review of clinical trials: a modest but easy step towards an accountable REB review structure in Canada. Health Law Rev 2005;13:39-50.

3. Alas JK, Godlovitch G, Mohan CM, et al. Regulatory framework for conducting clinical research in Canada. Can J Neurol Sci 2017;44:469-74.

4. Canada's clinical trial infrastructure: a prescription for improved access to new medicines. Ottawa: The Standing Senate Committee on Social Affairs, Science and Technology; 2012.

5. Coleman $\mathrm{CH}$, Bouësseau MC. How do we know that research ethics committees are really working? The neglected role of outcomes assessment in research ethics review. BMC Med Ethics 2008;9:6.

6. Caligiuri M, Allen K, Buscher N, et al. A multisite study of performance drivers among institutional review boards. J Clin Trans/ Sci 2017;1:192-7.

7. Fost N, Levine RJ. The dysregulation of human subjects research. JAMA 2007; 298:2196-8.

8. Evans L, Morin K, Manzo J, et al. Canadian Clinical Trials Coordinating Centre (CCTCC) Research Ethics Board (REB) Accreditation Working Group (WG) Final Recommendations (FRs). Ottawa: Canadian Clinical Trials Coordinating Centre (CCTCC); 2017.

9. Strategy for patient-oriented research: streamlining of Health Research Ethics Review External Advisory committee [report for discussion]. Ottawa: Canadian Clinical Trials Coordinating Centre (CCTCC); 2013.

10. Nicholls SG, Hayes TP, Brehaut JC, et al. A scoping review of empirical research relating to quality and effectiveness of research ethics review. PLoS One 2015; 10:e0133639.

11. Abbott L, Grady C. A systematic review of the empirical literature evaluating IRBs: what we know and what we still need to learn. J Empir Res Hum Res Ethics 2011;6:3-19.

12. Vulcano DM. Frustrations in benchmarking IRBs: reflections after analyzing the United States' IRB registration database. J Empir Res Hum Res Ethics 2012; 7:34-6. 
Competing interests: Laurel Evans was chair and Karine Morin was co-chair of the Canadian Clinical Trials Coordinating Centre (CCTCC) Research Ethics Board (REB) Accreditation Working Group. Stuart Nicholls was a member of the working group, and Holly Longstaff served as a paid consultant for the working group through her consulting firm Engage Associates. The views expressed within the manuscript do not represent the views of the authors' institutions or the views of the CCTCC.

This article has been peer reviewed.
Affiliations: Ottawa Hospital Research Institute (Nicholls); School of Epidemiology and Public Health (Nicholls), University of Ottawa, Ottawa, Ont.; independent consultant (Morin), Ottawa, Ont.; Office of Research Ethics (Evans), The University of British Columbia; Engage Associates Consulting Group (Longstaff), Vancouver, BC

Contributors: Stuart Nicholls drafted the manuscript. All of the authors made substantial contributions to the conception of the work, revised it critically for intellectual content and approved the final version to be published. All of the authors agree to be accountable for all aspects of the work.

Acknowledgements: The authors thank Diane Laflamme, Charles Weijer, Spencer Hey, Alex London, and Cory Goldstein for comments on sections of earlier versions of the manuscript.

Correspondence to: Stuart Nicholls, SNicholls@ohri.ca, snicholl@uottawa.ca 